\title{
Laboratórios Online Móveis em um Ambiente de Experimentação Colaborativo
}

\author{
José Pedro Schardosim Simão ${ }^{1}$, João Paulo Cardoso de Lima ${ }^{2}$, \\ Hamadou Saliah-Hassane $^{3}$, Juarez Bento da Silva ${ }^{1}$, João Bosco da Mota Alves ${ }^{1}$ \\ ${ }^{1}$ Laboratório de Experimentação Remota (RExLab) - \\ Universidadade Federal de Santa Catarina - Campus Araranguá (UFSC) \\ Araranguá/SC - Brasil \\ ${ }^{2}$ Instituto de Informática - Universidade Federal do Rio Grande do Sul (UFRGS) \\ Porto Alegre/RS - Brasil \\ ${ }^{3}$ Departamento de Ciência e Tecnologia - Téle-Université, Universidade de Quebec (TÉLUQ) \\ Montreal - Canada

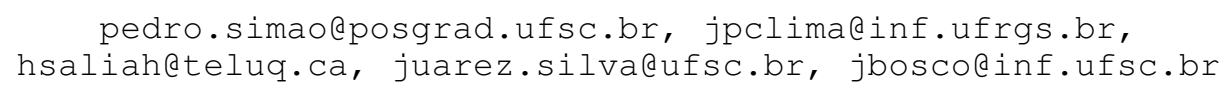

\begin{abstract}
Remote laboratories play an important role in increasing flexibility and the extent of practical activities in teaching. However, the current remote laboratories distribution model does not consider a dynamic scenario that includes collaboration, peer-to-peer labs and mobile labs. This paper presents a set of tools for creating collaborative learning environments with on-line mobile labs that allow students to implement their own laboratories and share them with classmates and teachers. The approach used is compatible with the machine and network configurations that the target user has in schools and at home, and provides the retrieval of information for learning evaluation.
\end{abstract}

Resumo. Os laboratórios remotos têm papel importante no aumento da flexibilização e do grau de alcance de atividades práticas no ensino. No entanto, o modelo atual de distribuição dos laboratórios remotos não considera um cenário dinâmico que inclua colaboração, laboratórios peer-to-peer e móveis. Neste artigo é apresentado um conjunto de ferramentas para criação de ambientes colaborativos de aprendizagem com laboratórios online móveis que permite aos estudantes criarem seus próprios laboratórios móveis e compartilhá-los com colegas e professores. A abordagem utilizada mostra-se compativel com configurações máquinas e redes que o usuário alvo possui em escolas e na sua residência e prevê a recuperação de informações para avaliação de aprendizagem.

\section{Introdução}

A experimentação é uma atividade primordial no ensino e aprendizagem de ciência, engenharia e tecnologia, e a realidade das práticas laboratoriais foi profundamente alterada pelo advento das Novas Tecnologias de Informação e Comunicação. Ferramentas como simulações, realidade virtual e laboratórios remotos têm sido usadas para tornar as aulas mais interativas. Mesmo os laboratórios tradicionais são, na maioria das vezes, mediados por computadores e outros dispositivos digitais. 
Diante do aumento significativo da matrícula em instituições de ensino e pesquisa em alguns países em desenvolvimento como o Brasil [UNESCO 2016], e com a necessidade de mobilidade de estudantes em alguns países desenvolvidos, grandes desafios precisam ser superados. Dentre as barreiras que já conhecemos, estão a disponibilidade e gestão de laboratórios convencionais para muitos alunos, aquisição e manutenção de equipamentos, bem como as atualizações de software que estão em constante mudança.

Nesse contexto, os laboratórios online têm sido usados como uma alternativa aos laboratórios tradicionais há alguns anos. Os laboratórios online são "ferramentas tecnológicas que um aluno pode utilizar para realizar trabalhos de laboratório em redes de computadores, sozinho ou em colaboração com outros participantes, a distância ou em um contexto de aprendizagem invertida"[Saliah-Hassane and Reuzeau 2014]. Os laboratórios remotos, por exemplo, têm sido usados para tornar as atividades de laboratório mais flexíveis em muitos ramos do conhecimento e em diferentes estágios educacionais: primário, secundário e terciário.

As simulações, também chamadas de laboratórios virtuais, seguem um modelo matemático para obter resultados próximos da natureza, mas não são capazes de lidar com o comportamento experimental em ambientes reais. Laboratórios remotos, por outro lado, usam dispositivos reais que podem ser manipulados pelos alunos, representando sistemas reais de forma mais eficaz.

A fim de implementar laboratórios remotos para o ensino e aprendizagem em ciências e engenharia, muitos desafios foram superados até agora, mas outras soluções ainda precisam ser encontradas. Por exemplo, a interoperabilidade e o gerenciamento de recursos federados de laboratórios remotos são uma grande preocupação quando se trata de compartilhar laboratórios entre diferentes instituições [Orduña 2013].

Como alternativa aos laboratórios remotos convencionais, neste artigo propomos o uso de laboratórios móveis por meio de um ambiente colaborativo. Neste cenário, os alunos criam seus próprios experimentos e compartilham o acesso e controle do dispositivo por meio de uma solução web, onde o professor tem acesso às interações realizadas e resultados obtidos.

\section{Experimentação em Ambientes Colaborativos}

Os laboratórios online têm sido frequentemente utilizados como complemento à experimentação prática, ou em alguns casos, como em cursos de educação a distância, como alternativa às atividades laboratoriais tradicionais. Esses recursos tecnológicos permitem que alunos e professores conduzam práticas laboratoriais de forma mais flexível e interativa, e podem oferecer experiências diferentes considerando as várias configurações possíveis.

Dependendo da localização e da natureza da experiência (quão real ou virtual ela é), os laboratórios online são geralmente classificados como virtuais, remotos ou híbridos, conforme modelo apresentado na Figura 1, adaptada de [Auer et al. 2003], [Zutin et al. 2010] e [Rodriguez-Gil et al. 2016].

Os laboratórios virtuais utilizam modelos matemáticos para simular os fenômenos estudados, compreendendo geralmente uma interface digital inspirada no equipamento real para apresentar entradas e saídas simuladas. Estes laboratórios são extremamente 
Figura 1. Figura

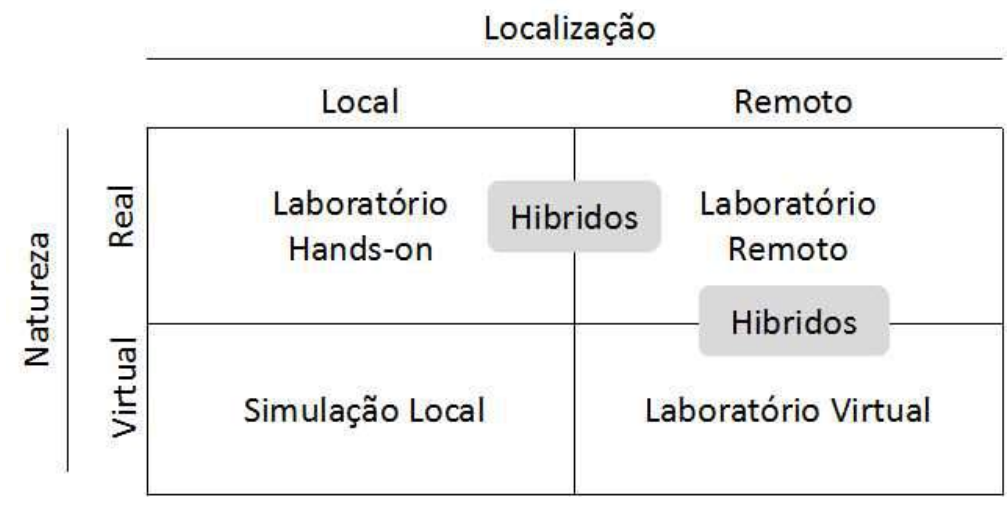

Figura 2. Classificação de laboratórios de acordo com a localização e a natureza

flexíveis e escaláveis, mas muitas vezes desconsideram variáveis de ambiente e erro experimental.

Os laboratórios remotos, por outro lado, baseiam-se em equipamentos reais que podem ser controlados pela Internet. No entanto, eles são muito mais difíceis de gerenciar e dimensionar, exigindo controle de concorrência ao recurso e a criação de múltiplas instâncias de um laboratório, a fim de apoiar a sua utilização em um ambiente de produção. Além disso, dependendo da configuração, um laboratório remoto pode ser muito específico, exigindo muitas regras de segurança para evitar danos [Rodriguez-Gil et al. 2016] e para garantir que o usuário obtenha os resultados esperados.

Laboratórios híbridos são soluções compostas por diferentes tipos de laboratórios. Geralmente, a abordagem para laboratórios híbridos considera somente casos compostos por laboratórios reais e virtuais, conforme descrito em [Bochicchio et al. 2015]. No entanto, dependendo do cenário de aprendizagem, um laboratório remoto poderia ser manipulado usando controles reais locais, como o uso de dispositivos hápticos apresentado por [Restivo et al. 2011], por exemplo, sendo então um laboratório híbrido.

O conceito em torno de laboratórios móveis tem sido explorado há algum tempo e interpretado de diferentes maneiras por pesquisadores em educação, ciência e engenharia [Costa and Alves 2006]. Uma das abordagens recorrentes, apresentada por [Silva et al. 2014] e [Zappatore et al. 2016], está associado com a concepção tradicional de experimentação remota, fazendo uso de dispositivos móveis para acessar e manipular laboratórios em diferentes locais através da internet.

Por outro lado, a ideia de laboratórios móveis apresentada neste trabalho considera o dispositivo que é objeto de experimentação sob controle móvel e hospedado pelo aluno. Assim, um aluno pode criar seu próprio laboratório usando microcontroladores de baixo custo, como Arduino, ou single-board computers, como Raspberry Pi. Mesmo que os laboratórios neste caso sejam reais e estejam em posse de um aluno, o dispositivo sob controle precisa estar conectado à Internet para compartilhar seu controle, entradas e saídas entre os usuários em um cenário colaborativo. Neste caso, estes laboratórios podem ser descritos como híbridos, uma vez que pode ser remoto ou hands-on dependendo de quem detém o equipamento e quem está o controlando. 
Além disso, os laboratórios móveis podem abrir uma série de novas possibilidades para ambientes colaborativos. Alguns avanços já foram feitos em pesquisas anteriores, a fim de enriquecer a colaboração na experimentação online, e soluções como ambientes colaborativos [Bochicchio et al. 2015] e mundos virtuais 3D foram utilizados para compartilhar laboratórios remotos[Antonio et al. 2016]. Usando os laboratórios móveis em tais cenários, vários grupos são capazes de realizar a mesma experiência ao mesmo tempo, os usuários podem pausar sessões e retomar a qualquer momento sem redefinir o laboratório ou aguardar outros usuários.

A fim de desenvolver uma camada de aplicação comum para laboratórios móveis, compatível com diferentes plataformas e clientes, é importante tomar uma abordagem baseada em módulos que podem ser reutilizados em diferentes aplicações. O modelo conhecido como Lab como um Serviço (LaaS) propõe que os laboratórios online sejam entregues como um serviço capaz de trocar e usar informações de diferentes sistemas e serviços [Tawfik et al. 2014]. Este método baseia-se no desenvolvimento de laboratórios online como módulos de componentes independentes, a fim de facilitar a manutenção, reutilização e interoperabilidade.

Nesse modelo, a especificação de Smart Device descreve a comunicação e as interfaces entre cliente e servidor, fornecendo um conjunto de informações que podem ser usadas para fornecer serviços da Web para acessar sensores e atuadores [Salzmann et al. 2015].

\section{Experiência de Aprendizagem em Experimentação Online}

A avaliação dos resultados de aprendizagem dos alunos é crucial para a realização de atividades laboratoriais em ambientes online. Acompanhar o comportamento dos usuários em aplicativos de aprendizagem é importante não apenas para avaliação, mas também para descobrir padrões de aprendizagem e projetar melhores atividades.

A captura e análise de dados de aprendizagem já foram exploradas em aplicações de laboratórios remotos, primeiramente usando a análise de redes sociais para acompanhar a experiência dos alunos [Orduña et al. 2014] e, recentemente, usando a aprendizagem analítica para oferecer aos alunos social e auto-consciência através do processo de experimentação remota [Venant et al. 2016].

Considerando o cenário dos laboratórios móveis, onde a experiência de aprendizagem é centrada no aluno, o professor precisa ter um feedback adequado do desenvolvimento da atividade para determinar se o experimento teve o resultado esperado e se os alunos aprenderam as habilidades necessárias nesse cenário. A avaliação também é um problema quando se usam laboratórios em cursos de grandes turmas ou MOOCs, o que requer um esforço considerável e tempo de professores e tutores.

O uso da Experience API (xAPI), anteriormente conhecida como TinCan API, permite a fácil descoberta de comportamento de aprendizagem, tornando possível a formalização, armazenamento e recuperação de experiências de aprendizagem [Hamzah et al. 2015]. Os dados armazenados podem ser utilizados pelos professores para acompanhar o progresso dos alunos em disciplinas gerais ou em competências específicas, facilitando a sua avaliação e a avaliação do objeto educativo.

Uma experiência de aprendizado em xAPI é rastreada e formatada em uma ins- 
trução, na qual um ator executa uma ação em um objeto (ator + verbo + atividade + propriedades adicionais) [ADL 2017]. Esta declaração é então armazenada em um Learning Record Store (LRS), de onde ele pode ser posteriormente recuperado e analisado.

O LRS pode fornecer dados para outras aplicações, como Learning Management Systems (LMS), e também tem um mecanismo de relatório, permitindo a visualização de dados da combinação de ator, verbo e atividade ao longo do tempo.

\section{Solução Desenvolvida}

A partir da necessidade de ferramentas e de padrões para compartilhar laboratórios online móveis, nesse trabalho é descrito um protótipo de ambiente de aprendizagem e componentes que dão suporte aos laboratórios móveis e à colaboração. A solução é composta por quatro componentes principais: Smart Device, Lab Gateway, Learning Record Store (LRS)e Lab@Home, um ambiente de aprendizagem colaborativa.

O primeiro componente define as interfaces de comunicação entre o servidor de laboratório e o cliente de laboratório. Para solucionar alguns problemas relacionados à configuração de rede e descoberta de dispositivos, um gateway é disposto entre o Lab@ Home e o Smart Device. O Lab@Home compreende diferentes características de um ambiente de aprendizagem colaborativo e fornece acesso a laboratórios remotos. Finalmente, a interação dos alunos com o ambiente de aprendizagem é armazenada para posterior análise, que é capturada pelo plugin xAPI no Lab@Home e registrada no LRS, conforme a Figura 2.

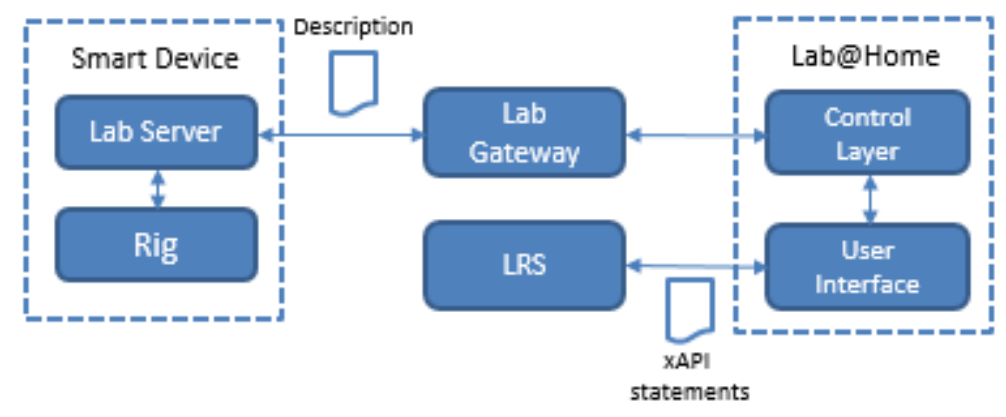

Figura 3. Arquitetura da solução

\subsection{Smart Device}

Neste cenário, o Smart Device, também chamado de textitlab server, é o servidor capaz de gerir um equipamento físico alvo, enquanto seu cliente, também conhecido como textitlab client, é a aplicação inclusa na interface do usuário do componente Lab@Home. A especificação de Smart Device implica em fornecer todas as funções e componentes de laboratório em um arquivo de descrição de serviços como um conjunto de serviços abstratos [Salzmann et al. 2015]. Nesse trabalho, a especificação é estendida para que os serviços do Smart Device não sejam barrados por firewalls, NAT ou por outra configuração de rede que poderia obstruir o acesso ao laboratório.

Esta abordagem baseia-se em um servidor de laboratório que atua como uma aplicação cliente usando protocolo WebSocket. Como o WebSocket fornece um canal de 
comunicação full-duplex sobre uma única conexão TCP, um cliente pode atuar como servidor e cliente ao mesmo tempo, semelhantemente ao princípio utilizado no WebRTC. Para esse comportamento, um gateway, chamado de Lab Gateway, é necessário para centralizar o acesso a esses servidores de laboratório.

Assim, uma aplicação cliente se conecta ao Lab Gateway e realiza as solicitações conforme o arquivo de descrição, que inclui solicitações HTTP e WebSocket. Foi adotada essa abordagem em vez de usar a placa como o servidor de laboratório porque neste cenário, onde o aluno pode usar o laboratório em casa, geralmente não há endereços IP válidos disponíveis.

Foram implementados dispositivos inteligentes utilizando duas plataformas de hardware, uma para dispositivos Arduino e compatíveis, e outra para single-board computers usando Node.js, como Raspberry Pi. Ambas as implementações são baseadas no protocolo Socket.IO, que estende os recursos do WebSocket.

Assim, usando esses códigos como exemplos, o equipamento e o servidor de laboratório podem ser desenvolvidos pelos próprios alunos e, em seguida, conectados a um Lab Gateway. A análise de circuitos elétricos é um exemplo simples de uso, uma vez que a maioria das placas de desenvolvimento permitem a geração e aquisição de sinais.

\subsection{Lab Gateway}

O Lab Gateway é a aplicação responsável por encaminhar as solicitações do cliente para o servidor de laboratório. Ele age como um middleware traduzindo solicitações, tanto WebSocket como HTTP, provindas dos usuários ou até mesmo do servidor de laboratório.

A comunicação tem de ser iniciada pelo servidor de laboratório, uma vez que ele é implementado como um cliente WebSocket. No entanto, esse estágio inicial também é usado para informar o estado real do laboratório, isto é, se está online ou offline. Além de enviar o arquivo de descrição, o laboratório também estabelece um canal WebSocket que será usado para trocar dados provenientes dos serviços de stub.

Assim que o arquivo de descrição é armazenado e analisado, o Lab Gateway cria um esboço $(s t u b)$ dos serviços do dispositivo inteligente e vincula as mensagens HTTP ou WebSocket provenientes dos usuários para esse servidor de laboratório. O Lab Gateway fornece a descrição do laboratório para o cliente de laboratório como se fosse um dispositivo inteligente, mesmo substituindo endereços e caminhos no arquivo de descrição do serviço.

O grupo de usuários que se conectará ao servidor de laboratório é definido no colaboratório, pois o gateway de laboratório compartilha informações através do mesmo banco de dados MySQL que o Lab@Home usa. Os usuários podem receber mensagens de broadcast originadas do servidor de laboratório e usar o mecanismo de controle de concorrência definido pelo servidor de laboratório, como fila e agendamento.

\subsection{Lab@Home}

O Lab@Home, o aplicativo cliente (Figura 3), foi desenvolvido como um aplicativo web responsivo, tornando-o compatível com dispositivos móveis e desktop. A interface do usuário usa HTML5, front-end Bootstrap 3 e a biblioteca JavaScript jQuery 3. O backend é baseado no Laravel 5.3, um framework PHP MVC. Para o bate-papo e compartilha- 
mento de arquivos, bem como para atualizar o status dos usuários, foi usado um servidor Node.JS com a biblioteca Socket.IO.

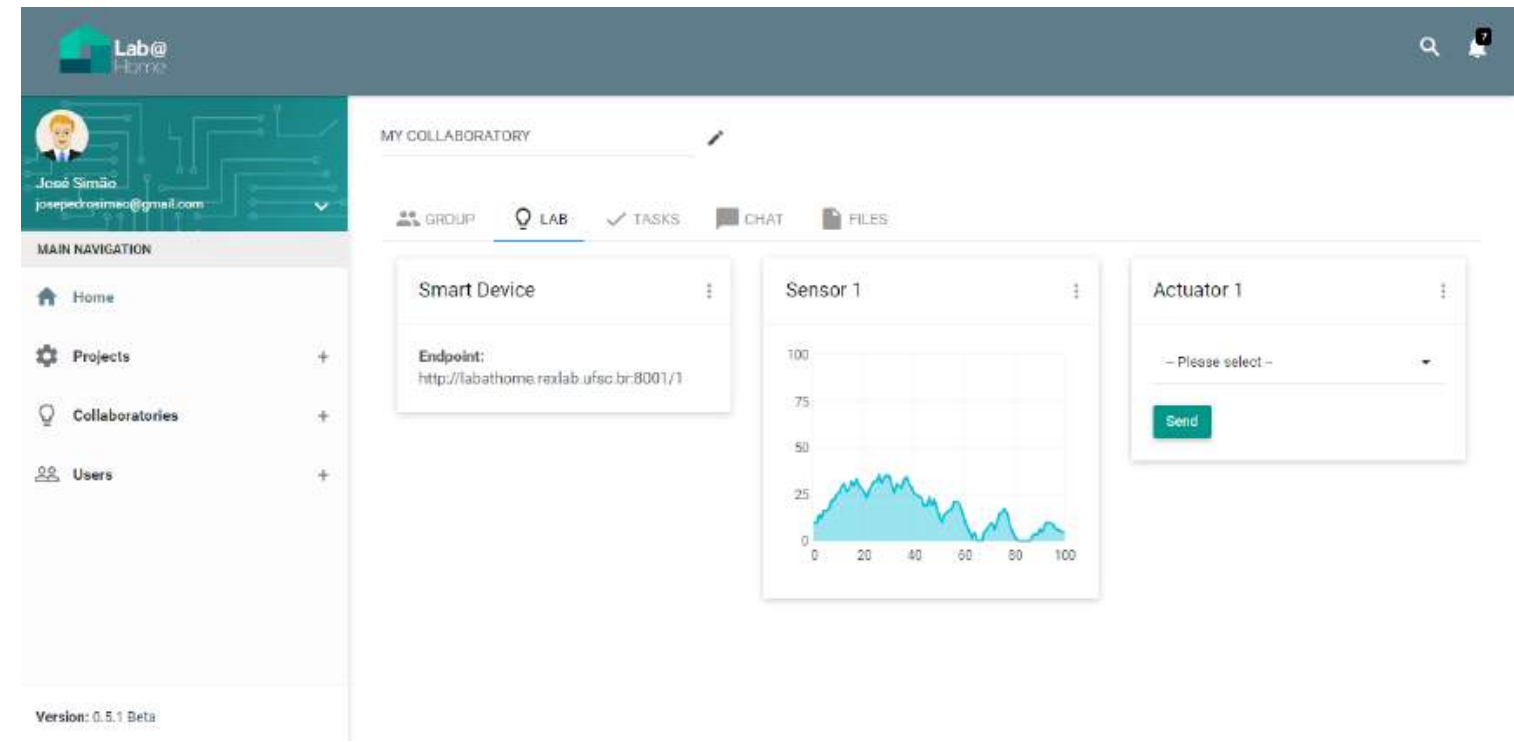

Figura 4. Exemplo de colaboratório

Os alunos acessam e manipulam os laboratórios, criam colaboratórios (ambientes de colaboração) para compartilhar dispositivos e interagir com outros usuários durante o processo de experimentação. Os espaços de trabalho são criados pelos próprios alunos, que também podem editar informações e adicionar outros membros.

Entre as funcionalidades disponíveis nos colaboratórios estão o gerenciamento de usuários (status do usuário, adição e remoção), compartilhamento de arquivos em repositórios de arquivos individuais e em grupo, sala de bate-papo e gerenciamento de tarefas (criação, atribuição, conclusão e devida). Os usuários também podem criar uma sala de videoconferência dentro do colaboratório, onde eles podem falar, compartilhar suas telas e mostrar o dispositivo em teste. A guia videoconferência foi desenvolvida usando Appear.in SDK, uma aplicação de vídeo chamada.

Todas as mensagens enviadas no chat, arquivos e informações de uma sessão são armazenadas no banco de dados, de modo que os usuários podem pausar e reiniciar uma sessão, ou recuperar dados de colaboradores terminados.

Os colaboratórios também podem ser criados a partir de modelos de projeto definidos por professores, com tarefas e arquivos específicos. Uma interface específica para o laboratório também pode ser definida pelo professor na criação do projeto, por isso, em uma tarefa, por exemplo, todos os alunos de um curso podem ter os mesmos recursos.

As ações e resultados de colaboratórios são armazenados em um LRS usando xAPI. Os usuários e dispositivos inteligentes são considerados como atores, armazenando não apenas as interações dos usuários com o sistema e entre si, mas também as mensagens enviadas pelo Smart Device. O vocabulário usado pelos atores humanos pode ser generalizado, e está mais ligado aos aspectos comportamentais (display, require, present, connect, disconnect, share, etc.), enquanto o vocabulário usado no smart device pode variar dependendo do experimento, sendo neste caso especificado no arquivo de descrição. 
Usamos uma instância do Learning Locker (Figura 4), um LRS baseado em PHP que recebe as instruções geradas na interface do usuário colaborador por solicitações AJAX. Esta implementação fornece funcionalidades de relatório, tornando possível gerar gráficos e exportar dados por diferentes combinações de atores, verbos e objetos.

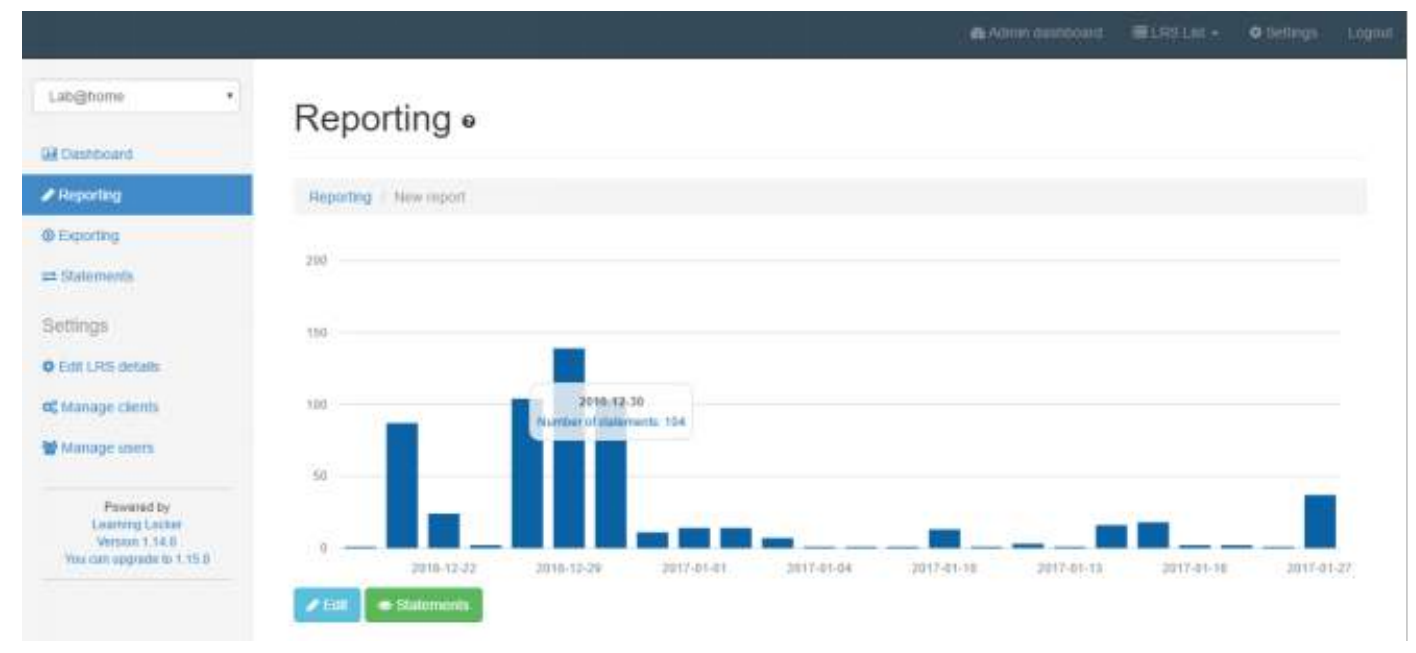

Figura 5. Learning Record Store

\section{Considerações Finais}

Este trabalho abordou a criação de um ambiente colaborativo para a experimentação online móvel. A solução possibilita que os alunos criem seus próprios laboratórios e os compartilhem em um ambiente colaborativo. Além disso, fornecemos modelos de software para placas de desenvolvimento amplamente conhecidas, o que permite que os alunos usem seus próprios equipamentos eletrônicos para ampliar o trabalho de laboratório usando a solução desenvolvida.

O modelo adotado no desenvolvimento, usando o gateway de laboratório para conectar dispositivos sem endereços IP válidos ao aplicativo cliente, pode ser usado em diferentes cenários para publicar laboratórios online, minimizando problemas com NAT e firewalls.

Em relação ao uso de xAPI, ainda não há vocabulário especifico para armazenar experiências de aprendizado de laboratórios online. Padronizado este vocabulário é de extrema importância, permitindo a cooperação e comparação de resultados entre diferentes estudos. Nesse sentido, como um trabalho futuro, seria importante definir verbos e atividades para o uso de XAPI com laboratórios online.

\section{Agradecimentos}

Os autores gostariam de agradecer ao Departamento de Relações Exteriores, Comércio e Desenvolvimento (DFTAD) do Governo do Canadá pela bolsa concedida através do Programa Futuros Líderes nas Américas (Emerging Leaders in the Americas Program, ELAP) e ao Conselho Nacional de Desenvolvimento Científico e Tecnológico (CNPq) pelo apoio financeiro através de bolsas e materiais para condução da pesquisa. 


\section{Referências}

ADL, A. D. L. I. (2017). Experience API: Advanced Distributed Learning (ADL) CoLaboratories.

Antonio, C. P., Lima, J. P. C., Alves, J. B. M., Silva, J. B., and Simão, J. P. S. (2016). Merging a remote microscope and virtual worlds: Teaching kingdom plantae on basic education. International Journal of Online Engineering (iJOE), 12(4):3.

Auer, M., Pester, A., Ursutiu, D., and Samoila, C. (2003). Distributed virtual and remote labs in engineering. In IEEE International Conference on Industrial Technology, 2003, volume 2, pages 1208-1213.

Bochicchio, M. A., Longo, A., Vaira, L., and Zappatore, M. (2015). Fostering online scientific experimentations in universities and high schools: The edoc project. In 2015 3rd Experiment International Conference, pages 337-342.

Costa, R. J. and Alves, G. R. (2006). Remote and mobile experimentation: Pushing the boundaries of an ubiquitous learning place. IFAC Proceedings Volumes, 39(4):314 320 .

Hamzah, W. M. A. F. W., Ali, N. H., Saman, M. Y. M., Yusoff, M. H., and Yacob, A. (2015). The use of tin can api for web usage mining in e-learning applications on the social network. In 2015 IEEE Symposium on Computer Applications and Industrial Electronics (ISCAIE), pages 113-118.

Orduña, P. (2013). Transitive and Scalable Federation Model for Remote Laboratories. Thesis, Universidad de Deusto.

Orduña, P., Almeida, A., López-de Ipiña, D., and Garcia-Zubia, J. (2014). Learning analytics on federated remote laboratories: Tips and techniques. In 2014 IEEE Global Engineering Education Conference (EDUCON), pages 299-305.

Restivo, M. T., Lopes, A. M., Machado, L. d. S., and Moraes, R. M. d. (2011). Adding tactile information to remote and virtual labs. In 2011 IEEE Global Engineering Education Conference, pages 1120-1124.

Rodriguez-Gil, L., Garcia-Zubia, J., Orduna, P., and Lopez-de Ipina, D. (2016). Towards new multiplatform hybrid online laboratory models. IEEE Transactions on Learning Technologies, PP(99):1-1.

Saliah-Hassane, H. and Reuzeau, A. (2014). Mobile open online laboratories: A way towards connectionist massive online laboratories with x-api (c-mools). In 2014 IEEE Frontiers in Education Conference (FIE) Proceedings, pages 1-7.

Salzmann, C., Govaerts, S., Halimi, W., and Gillet, D. (2015). The smart device specification for remote labs. International Journal of Online Engineering (iJOE), 11(4):9-29.

Silva, J. B. d., Rochadel, W., Simão, J. P. S., and Fidalgo, A. V. d. S. (2014). Adaptation model of mobile remote experimentation for elementary schools. IEEE Revista Iberoamericana de Tecnologias del Aprendizaje, 9(1):28-32.

Tawfik, M., Salzmann, C., Gillet, D., Lowe, D., Saliah-Hassane, H., Sancristobal, E., and Castro, M. (2014). Laboratory as a service (laas): a novel paradigm for developing and implementing modular remote laboratories. International Journal of Online Engineering (iJOE), 10(4):9. 
UNESCO, I. f. S. (2016). Brazil - education and literacy.

Venant, R., Vidal, P., and Broisin, J. (2016). Learning analytics for learner awareness in remote laboratories dedicated to computer education. In Workshop on Learning Analytics for Learners in 6th International Conference on Learning Analytics and Knowledge (LAK 2016), pages 31-37, New York, NY, USA. ACM.

Zappatore, M., Longo, A., and Bochicchio, M. A. (2016). Enabling mool in acoustics by mobile crowd-sensing paradigm. In 2016 IEEE Global Engineering Education Conference (EDUCON), pages 733-740.

Zutin, D. G., Auer, M. E., Maier, C., and Niederstätter, M. (2010). Lab2go: A repository to locate educational online laboratories. In IEEE EDUCON 2010 Conference, pages 1741-1746. 